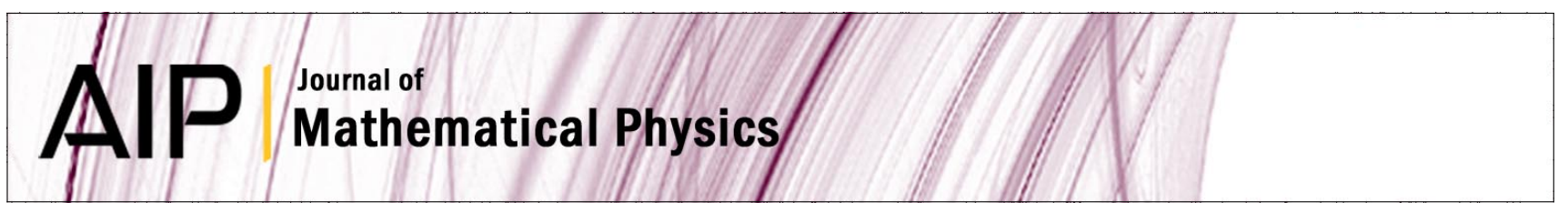

\title{
On form-preserving transformations for the time-dependent Schrödinger equation
}

Federico Finkel, Artemio González-López, Niky Kamran, and Miguel A. Rodríguez

Citation: J. Math. Phys. 40, 3268 (1999); doi: 10.1063/1.532885

View online: http://dx.doi.org/10.1063/1.532885

View Table of Contents: http://jmp.aip.org/resource/1/JMAPAQ/v40/i7

Published by the American Institute of Physics.

\section{Additional information on J. Math. Phys.}

Journal Homepage: http://jmp.aip.org/

Journal Information: http://jmp.aip.org/about/about_the_journal

Top downloads: http://jmp.aip.org/features/most_downloaded

Information for Authors: http://jmp.aip.org/authors

\section{ADVERTISEMENT}

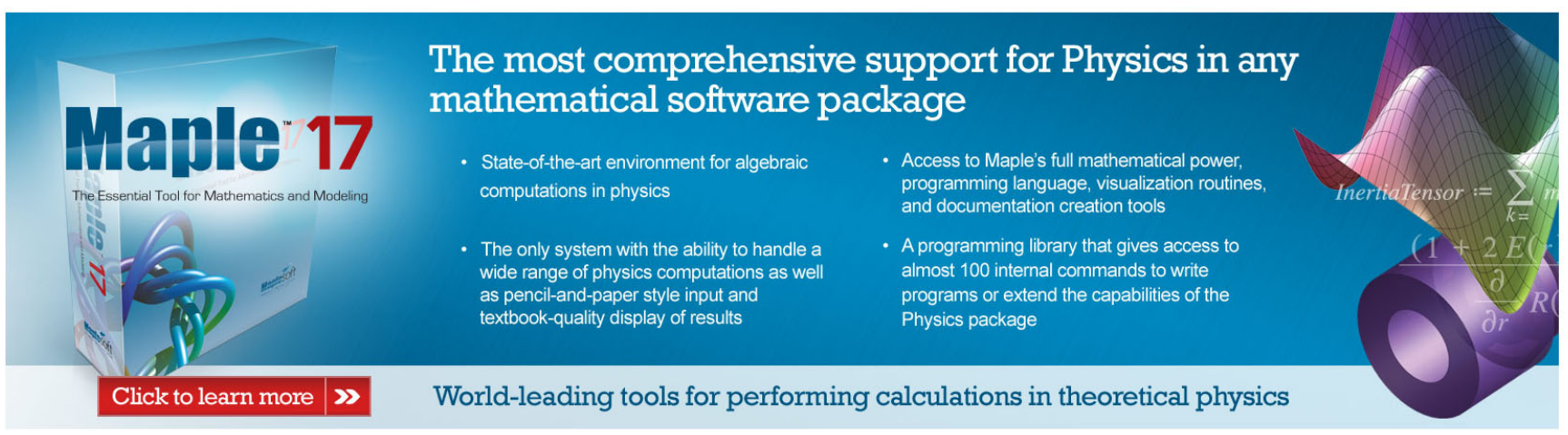




\title{
On form-preserving transformations for the time-dependent Schrödinger equation
}

\author{
Federico Finkel and Artemio González-López \\ Departamento de Física Teórica II, Universidad Complutense de Madrid, \\ E-28040 Madrid, Spain \\ Niky Kamran \\ Department of Mathematics and Statistics, McGill University, \\ Montréal, Québec H3A 2K6, Canada \\ Miguel A. Rodríguez \\ Departamento de Física Teórica II, Universidad Complutense de Madrid, \\ E-28040 Madrid, Spain
}

(Received 22 December 1998; accepted for publication 18 March 1999)

\begin{abstract}
In this paper we point out a close connection between the Darboux transformation and the group of point transformations which preserve the form of the timedependent Schrödinger equation (TDSE). In our main result, we prove that any pair of time-dependent real potentials related by a Darboux transformation for the TDSE may be transformed by a suitable point transformation into a pair of timeindependent potentials related by a usual Darboux transformation for the stationary Schrödinger equation. Thus, any (real) potential solvable via a time-dependent Darboux transformation can alternatively be solved by applying an appropriate form-preserving point transformation of the TDSE to a time-independent potential. The pre-eminent role of the latter type of transformations in the solution of the TDSE is illustrated with a family of quasi-exactly solvable time-dependent anharmonic potentials. (C) 1999 American Institute of Physics.
\end{abstract}

[S0022-2488(99)00207-8]

\section{INTRODUCTION}

A considerable amount of research has been devoted over the past few years to the exact solution of the time-dependent Schrödinger equation (TDSE) in $1+1$ dimensions. Several modifications of the celebrated Darboux transformation for the stationary Schrödinger equation, ${ }^{1,2}$ have been proposed in this respect in the literature. Matveev and Salle showed that the usual Darboux transformation for the stationary Schrödinger equation could also be applied to the TDSE with a time-dependent potential. ${ }^{3}$ An equivalent approach was followed by Bluman and Shtelen in Ref. 4, who considered a nonlocal transformation which is precisely the inverse map of the usual Darboux transformation. The Darboux transform of a time-dependent potential is in general a complexvalued function. (The explicit conditions for the resulting potential to be real-valued appear in a recent paper by Bagrov and Samsonov. ${ }^{5}$ ) For this reason, several generalizations of the Darboux transformation mapping real potentials to real potentials have been proposed in the literature. The best known of these generalizations is the binary Darboux transformation described in Ref. 3, which is in fact one of the main tools for finding exact solutions of integrable equations.

A seemingly unrelated method of constructing exact solutions of the TDSE which has proved remarkably successful is based on the use of point transformations which preserve the form of the TDSE. The idea goes back to the work of Leach on the time-dependent harmonic oscillator, ${ }^{6}$ arising, e.g., in the study of the motion of charged particles in a Paul trap. ${ }^{7}$ The method was subsequently extended by Bluman ${ }^{8,9}$ and Ray ${ }^{10}$ to obtain exact solutions of the TDSE for a quadratic potential with arbitrary time-dependent coefficients. The technique has also been applied to time-dependent harmonic oscillators with a repulsive barrier, ${ }^{11}$ and to anisotropic timedependent harmonic potentials in $2+1$ dimensions. $^{12}$ 
The main purpose of this paper is to characterize the most general time-dependent real potential whose Darboux transform is real. A partial result along these lines for potentials rapidly decreasing at spatial infinity was mentioned in Ref. 13. As noted in this reference, the latter potentials are of limited interest regarded as solutions of the KP equation. However, potentials of this type (and, more generally, of the type considered in this paper) are interesting from the point of view of exactly solving the time-dependent Schrödinger equation, as underscored by the recent work of Bagrov and Samsonov. ${ }^{5,14}$

In this paper we show that the Darboux transformation for the TDSE is in fact closely related to the point transformation method. To this end, in Sec. II we briefly review the Darboux transformation and the point transformations preserving the form of the TDSE, applying the latter to construct a time-dependent anharmonic oscillator potential admitting a certain number of algebraically computable wave functions. In Sec. III we derive the main results of our paper, proving that any time-dependent real-valued potential for which the Darboux transformation yields a real potential may always be mapped to a time-independent potential by a form-preserving point transformation of the TDSE. Moreover, the Darboux transformation for any such potential is equivalent to a Darboux transformation for its associated time-independent potential, followed by the inverse of the corresponding point transformation. Finally, Sec. IV is devoted to our concluding remarks and related open questions.

\section{GENERAL BACKGROUND}

In this section we summarize the basics of the Darboux and the form-preserving point transformations for the TDSE. Following Bagrov and Samsonov, ${ }^{5}$ we take as the starting point of the Darboux transformation for the TDSE the intertwining relation

$$
L\left(i \partial_{t}-H_{0}\right)=\left(i \partial_{t}-H_{1}\right) L,
$$

where

$$
H_{i}=-\partial_{x}^{2}+V_{i}(x, t), \quad i=0,1,
$$

and $L$ is a first-order differential operator of the form

$$
L=L_{1}(x, t) \partial_{x}+L_{0}(x, t) .
$$

It follows immediately from the intertwining relation (1) that if $\psi_{0}$ solves the TDSE with Hamiltonian $H_{0}$, then $\psi_{1}=L \psi_{0}$ will solve the TDSE with Hamiltonian $H_{1}$. It is also easily verified that the intertwining relation (1) will be satisfied if and only if

$$
L=L_{1} \cdot\left(\partial_{x}+\chi_{x}\right), \quad V_{1}=V_{0}+2 \chi_{x x}+i\left(\log L_{1}\right)_{t},
$$

where $e^{-\chi}$ is a solution of the TDSE with the potential $V_{0}$, and $L_{1}=L_{1}(t)$ is an arbitrary function. The transformed potential $V_{1}(x, t)$ is a real-valued function if and only if

$$
\operatorname{Im} \chi_{x x x}=0
$$

and

$$
\left|L_{1}\right|=\exp \left[-2 \int_{t_{0}}^{t} \operatorname{Im} \chi_{x x}(x, s) d s\right] .
$$

Without loss of generality, we shall assume from now on that $L_{1}$ is real and positive, and is therefore given by the right-hand side of (5).

Just as in the time-independent case, the Darboux transformation for the TDSE can be inverted. Indeed, if $\psi_{1}$ is a solution of the TDSE with potential $V_{1}$ given by (3), the function 


$$
\psi_{0}(x, t)=\frac{e^{-\chi(x, t)}}{L_{1}(t)}\left[\int_{x_{0}}^{x} e^{\chi(y, t)} \psi_{1}(y, t) d y+c_{0}(t)\right]
$$

with $c_{0}(t)$ given by

$$
c_{0}(t)=i L_{1}(t) \int_{t_{0}}^{t} \frac{e^{\chi\left(x_{0}, s\right)}}{L_{1}(s)}\left(\psi_{1, x}\left(x_{0}, s\right)-\chi_{x}\left(x_{0}, s\right) \psi_{1}\left(x_{0}, s\right)\right) d s
$$

solves the TDSE with potential $V_{0}$. If the factor $L_{1}$ is taken as unity, the mapping $\psi_{1} \mapsto \psi_{0}$ given by (6) reduces to the nonlocal transformation considered by Bluman and Shtelen in Ref. 4.

The most general point transformation mapping the TDSE

$$
\left(i \partial_{t}+\partial_{x}^{2}-V_{0}(x, t)\right) \psi_{0}(x, t)=0
$$

for any given potential $V_{0}$ into another TDSE with potential $\bar{V}_{0}$ for the transformed wave function $\bar{\psi}_{0}$ is defined by

$$
\begin{gathered}
\bar{x}=\frac{x}{C(t)}+B(t), \quad \bar{t}=\int_{t_{0}}^{t} \frac{d s}{C^{2}(s)}, \\
\psi_{0}(x, t)=|C|^{-1 / 2} \exp \left[\frac{i}{4}\left(\frac{\dot{C}}{C} x^{2}-2 \dot{B} C x+A(t)\right)\right] \bar{\psi}_{0}(\bar{x}, \bar{t}), \\
V_{0}(x, t)=\frac{1}{C^{2}} \bar{V}_{0}(\bar{x}, \bar{t})-\frac{\ddot{C}}{4 C} x^{2}+\left(\frac{C \ddot{B}}{2}+\dot{B} \dot{C}\right) x-\frac{1}{4}\left(C^{2} \dot{B}^{2}+\dot{A}\right),
\end{gathered}
$$

where $A, B$, and $C \neq 0$ are real-valued functions of $t$. Note that square-integrability is preserved under the transformation (8). As remarked before, the point transformation (8) has been employed to construct exact solutions of the TDSE for quadratic potentials with time-dependent coefficients. The interest of the transformation (8) is not limited, however, to quadratic (or exactly solvable) time-dependent potentials, as evidenced by the following example:

Example: Consider the two-parameter family of anharmonic oscillator potentials given by ${ }^{15-17}$

$$
\bar{V}(\bar{x})=\bar{x}^{6}+2 \alpha \bar{x}^{4}+\left(\alpha^{2}-4 n-3\right) \bar{x}^{2},
$$

where $\alpha \in \mathbb{R}$ and $n \in \mathbb{N}$. The sextic potential (9) is a well-known example of the class of quasiexactly solvable potentials, for which a certain subset of the spectrum can be computed by purely algebraic means; see Ref. 18 for an extensive review of the field. The first $n+1$ even bound states of the potential (9) are of the form

$$
\phi(\bar{x})=\exp \left[-\frac{1}{4} \bar{x}^{4}-\frac{\alpha}{2} \bar{x}^{2}\right] p\left(\bar{x}^{2}\right),
$$

where $p(s)$ is a polynomial in $s$ of degree less than or equal to $n$ which can be computed algebraically. The point transformation (8) with $A=B=0$ and $C=\omega^{-1 / 2}, \omega=\omega(t)$ being a positive function, leads directly to the potential $V(x, t)$ given by

$$
V(x, t)=\omega^{4} x^{6}+2 \alpha \omega^{3} x^{4}+\left(\alpha^{2}-4 n-3-\frac{3 \dot{\omega}^{2}-2 \omega \ddot{\omega}}{16 \omega^{4}}\right) \omega^{2} x^{2} .
$$

The TDSE with potential (11) possesses $n+1$ square-integrable solutions of the form 


$$
\psi(x, t)=\omega^{1 / 4} \exp \left[-i\left(\frac{\dot{\omega}}{8 \omega} x^{2}+E \int_{t_{0}}^{t} \omega(s) d s\right)\right] \phi(\sqrt{\omega} x),
$$

where $\phi(\bar{x})$ is an algebraic eigenfunction of the form (10) with eigenvalue $E$ of the Hamiltonian

$$
\bar{H}=-\partial_{\bar{x}}^{2}+\bar{V}(\bar{x}) .
$$

The potential (11) thus provides a natural extension of the notion of quasi-exact solvability to the time-dependent case, in the sense that the associated TDSE admits a certain number of solutions which can be determined algebraically. In particular, note that if $\omega(t)$ is of the form

$$
\omega_{0}(t)=\beta\left[\gamma+\left(\gamma^{2}-\left(\alpha^{2}-4 n-3\right) \beta\right)^{1 / 2} \sin (4 \sqrt{\beta} t+\delta)\right]^{-1},
$$

with $\delta \in \mathbb{R}, \beta>0$ and $\gamma^{2}>\left(\alpha^{2}-4 n-3\right) \beta>0$, the potential (11) reduces to a harmonic oscillator with a periodic-in-time anharmonic perturbation, namely,

$$
V_{0}(x, t)=\omega_{0}^{4}(t) x^{6}+2 \alpha \omega_{0}^{3}(t) x^{4}+\beta x^{2} .
$$

\section{THE REALITY CONDITION AND THE FORM-PRESERVING POINT TRANSFORMATIONS}

In this section we prove the main results of our paper, starting with the following theorem:

Theorem: Let $e^{-\chi}$ be a solution of the TDSE with potential $V_{0}(x, t)$. If $\chi$ satisfies the reality condition (4), then $V_{0}(x, t)$ may be mapped to a time-independent potential $\bar{V}_{0}(\bar{x})$ by a point transformation (8).

Proof: Let $\chi_{0}=\operatorname{Re} \chi, \chi_{1}=\operatorname{Im} \chi$. The TDSE for $e^{-\chi}$ is then equivalent to the pair of real equations given by

$$
\begin{gathered}
\chi_{0, t}+\chi_{1, x x}-2 \chi_{0, x} \chi_{1, x}=0, \\
V_{0}(x, t)=\chi_{1, t}-\chi_{0, x x}-\chi_{1, x}^{2}+\chi_{0, x}^{2} .
\end{gathered}
$$

If the reality condition (4) holds, i.e., if $\chi_{1}$ is of the form

$$
\chi_{1}=a(t) x^{2}+b(t) x+c(t),
$$

Eqs. (13) and (14) reduce to

$$
\begin{gathered}
\chi_{0, t}-2(2 a x+b) \chi_{0, x}+2 a=0, \\
V_{0}(x, t)=\left(\dot{a}-4 a^{2}\right) x^{2}+(\dot{b}-4 a b) x+\dot{c}-b^{2}+\chi_{0, x}^{2}-\chi_{0, x x} .
\end{gathered}
$$

The general solution of Eq. (16) is of the form ${ }^{19}$

$$
\chi_{0}=-2 \int_{t_{0}}^{t} a(s) d s+F\left(e^{4 \int_{t_{0}}^{t} a(s) d s} x+2 \int_{t_{0}}^{t} b(s) e^{4 \int_{t_{0}}^{s} a(r) d r} d s\right)
$$

where $F$ is an arbitrary real-valued function. Substituting this expression into (17), we immediately conclude that the transformation (8) determined by

$$
C(t)=e^{-4 \int_{t_{0}}^{t} a(s) d s}, \quad B(t)=2 \int_{t_{0}}^{t} \frac{b(s)}{C(s)} d s, \quad A(t)=-4 c(t),
$$

maps the potential $V_{0}(x, t)$ into the time-independent potential 


$$
\bar{V}_{0}(\bar{x})=F^{\prime 2}(\bar{x})-F^{\prime \prime}(\bar{x}) .
$$

Thus, any potential for which the Darboux transformation yields another real-valued potential may be mapped into a time-independent potential by a form-preserving point transformation. It is easy to check that the transform of $e^{-\chi}$ under the point transformation defined by (8), (15), (18), (19) is $e^{-F}$, which is therefore an eigenfunction of the time-independent potential $\bar{V}_{0}(\bar{x})$. The next Corollary shows that the usual Darboux transform of the associated time-independent potential generated by $F$ is related to the Darboux transform of the original potential by the same point transformation.

Corollary: Let $e^{-\chi}=e^{-\chi_{0}-i \chi_{1}}$ be a solution of the TDSE with potential $V_{0}(x, t)$, with $\chi$ satisfying the reality condition (4). Let $\mathcal{D}$ and $\mathcal{T}$ denote, respectively, the Darboux transformation (3) and the point transformation (8) defined by $\chi$ via Eqs. (15), (18), (19). Let $\overline{\mathcal{D}}$ denote the Darboux transformation generated by $F$. Then

$$
\mathcal{T} \circ \mathcal{D}=\overline{\mathcal{D}} \circ \mathcal{T}
$$

Remark: This result may be easily visualized with the help of the following commutative diagram:

$$
\begin{array}{ccc}
V_{0}(x, t) & \stackrel{\mathcal{T}}{\rightarrow} & \bar{V}_{0}(\bar{x})=F^{2}-F^{\prime \prime} \\
\mathcal{D} \downarrow & & \downarrow \overline{\mathcal{D}} \\
V_{1}(x, t) & \stackrel{\mathcal{T}}{\rightarrow} & \bar{V}_{1}(\bar{x})=F^{\prime 2}+F^{\prime \prime}
\end{array} .
$$

Proof: The proof follows from a straightforward application of the appropriate formulas for the transformed potentials and wave functions.

Q.E.D.

The above Corollary shows, in particular, that the potential $V_{1}(x, t)$ is the image under the inverse of the form-preserving point transformation $\mathcal{T}$ determined by (8), (15), (18), (19) of a time-independent potential $\bar{V}_{1}(\bar{x})$. Exact solutions of the TDSE with potential $V_{1}$ can therefore be obtained simply by applying the point transformation $\mathcal{T}^{-1}$ to solutions of the TDSE for the time-independent potential $\bar{V}_{1}$.

Another important consequence of the above Corollary is that, if the potential $\bar{V}_{0}$ satisfies (for instance) the condition

$$
\int_{-\infty}^{\infty}\left|\bar{V}_{0}(\bar{x})\right|(1+|\bar{x}|) d \bar{x}<\infty
$$

then the time-dependent Darboux transformation (3) preserves the square-integrability of eigenfunctions. Indeed, if $\bar{V}_{0}$ verifies (21) then the time-independent Darboux transformation $\overline{\mathcal{D}}$ determined by a nonvanishing eigenfunction of $\bar{V}_{0}$ preserves square integrability. ${ }^{20,21}$ The result stated above then follows easily from (20), the invertibility of $\mathcal{T}$, and the fact that the form-preserving point transformation $\mathcal{T}$ always preserves square integrability.

Example: It is straightforward to verify that all the examples of time-dependent potentials appearing in Refs. 4, 5, 14 which are solvable by means of a Darboux transformation are indeed the images of certain exactly solvable time-independent potentials under suitable form-preserving point transformations.

For instance, the free-particle potential $V_{0}(x, t)=0$ admits a one-parameter family of solutions

$$
\psi_{\lambda}(x, t)=\left(1+t^{2}\right)^{-1 / 4} \exp \left[\frac{i}{4}\left(\frac{t x^{2}}{1+t^{2}}+4 \lambda \arctan t\right)\right] Q_{\lambda}\left(x / \sqrt{1+t^{2}}\right)
$$

satisfying the reality condition (4), where $Q_{\lambda}$ is a (real-valued) solution of Weber's equation 


$$
Q_{\lambda}^{\prime \prime}(y)-\left(\frac{y^{2}}{4}+\lambda\right) Q_{\lambda}(y)=0
$$

(see Ref. 14, Eq. (18)). By the Theorem at the beginning of this section, it follows that $V_{0}$ is related to a certain time-independent potential $\bar{V}_{0}$ by a point transformation (8). Indeed, in this case we have

$$
\chi_{1}=-\operatorname{Im} \log \psi_{\lambda}=-\frac{t x^{2}}{4\left(1+t^{2}\right)}-\lambda \arctan t
$$

so from (19) it follows that

$$
C=\sqrt{1+t^{2}}, \quad B=0, \quad A=4 \lambda \arctan t .
$$

Substituting these formulas into (8) we find that

$$
\bar{x}=\frac{x}{\sqrt{1+t^{2}}}
$$

and

$$
\bar{V}_{0}(\bar{x})=\frac{\bar{x}^{2}}{4}+\lambda
$$

is a harmonic oscillator potential.

For all $n \in \mathbb{N}$, let $H_{n}$ denote the $n$th Hermite polynomial. The functions ${ }^{22}$

$$
Q_{n+1 / 2}(y)=i^{n} e^{y^{2} / 4} H_{n}(i y / \sqrt{2})
$$

are real-valued solutions of Weber's equation (22) with $\lambda=n+\frac{1}{2}$ without zeros on the positive real semiaxis. ${ }^{23}$ Hence, for all $n \in \mathbb{N}$ the Darboux transformation determined by the eigenfunction $\psi_{n+1 / 2}$ of $V_{0}$ is well-defined on the positive real semiaxis. From Eqs. (3), (5) and the definition of $\psi_{\lambda}$, it follows that the transformed potential $V_{1}$ is given by

$$
V_{1}(x, t)=2 \chi_{0, x x}=-2\left[\log Q_{n+1 / 2}(\bar{x})\right]_{x x}=\frac{2}{1+t^{2}}\left(\frac{Q_{n+1 / 2}^{\prime 2}(\bar{x})}{Q_{n+1 / 2}^{2}(\bar{x})}-\frac{\bar{x}^{2}}{4}-n-\frac{1}{2}\right),
$$

where $\bar{x}$ is given by (24). Using standard identities for the derivatives of the Hermite polynomials, the reader can easily verify that this formula for $V_{1}(x, t)$ agrees with the corresponding expression given in Ref. 14. As stated in the Corollary, the potential $V_{1}(x, t)$ is related by the point transformation (8) defined by (23) to a time-independent potential $\bar{V}_{1}(\bar{x})$ obtained from $\bar{V}_{0}(\bar{x})$ by applying a time-independent Darboux transformation $\overline{\mathcal{D}}$. From (18) and the definition of $\psi_{\lambda}$, it easily follows that the function $F(\bar{x})$ generating the Darboux transformation $\overline{\mathcal{D}}$ is given by

$$
F(\bar{x})=-\log Q_{n+1 / 2}(\bar{x}),
$$

and therefore

$$
\bar{V}_{1}(\bar{x})=F^{\prime 2}(\bar{x})+F^{\prime \prime}(\bar{x})=2 \frac{Q_{n+1 / 2}^{\prime 2}(\bar{x})}{Q_{n+1 / 2}^{2}(\bar{x})}-\frac{\bar{x}^{2}}{4}-n-\frac{1}{2} .
$$

It is straightforward to check that the potentials $V_{1}(x, t)$ and $\bar{V}_{1}(\bar{x})$ are indeed related by the point transformation (8) determined by (23). 


\section{CONCLUSIONS}

In this paper, we have shown that the Darboux transformation for the time-dependent Schrödinger equation is essentially equivalent to the usual Darboux transformation for the stationary Schrödinger equation. Any (real) potential $V_{1}(x, t)$ solvable via a time-dependent Darboux transformation starting from a real potential $V_{0}(x, t)$ can alternatively be solved by applying a formpreserving point transformation to a time-independent potential $\bar{V}_{1}(\bar{x})$. As a matter of fact, although a large number of methods and papers have been devoted in recent times to the exact solution of the TDSE, ${ }^{24}$ most of the associated time-dependent Hamiltonians either are not of the standard form (2), or are also obtainable from a time-independent Hamiltonian by a formpreserving point transformation.

The interest of the Darboux transformation for the TDSE as a useful method to obtain new (quasi-)exactly solvable time-dependent potentials is therefore very limited. It should be noted, however, that the Darboux transformation for the TDSE could still render helpful results if the starting potential is not a real-valued function but only the transformed potential is real. ${ }^{25}$ As a final remark, we would like to stress that the Darboux transformation may still be useful to construct exact solutions to real-valued diffusion equations of the Fokker-Planck-type, for which no reality condition as Eq. (4) must be considered.

\section{ACKNOWLEDGMENTS}

F.F., A.G.-L., and M.A.R. would like to acknowledge the partial financial support of the DGICYT under Grant No. PB95-0401. N.K. was supported in part by NSERC Grant No. 0GP0105490.

${ }^{1}$ G. Darboux, C. R. Acad. Sci. Paris 94, 1456 (1882).

${ }^{2}$ F. Cooper, A. Khare, and U. Sukhatme, Phys. Rep. 251, 267 (1995).

${ }^{3}$ V. B. Matveev and M. A. Salle, Darboux Transformations and Solitons (Springer, Berlin, 1991).

${ }^{4}$ G. Bluman and V. Shtelen, J. Phys. A 29, 4473 (1996).

${ }^{5}$ V. G. Bagrov and B. F. Samsonov, Phys. Lett. A 210, 60 (1996).

${ }^{6}$ P. G. L. Leach, J. Math. Phys. 18, 1902 (1977).

${ }^{7}$ L. S. Brown, Phys. Rev. Lett. 66, 527 (1991).

${ }^{8}$ G. Bluman, SIAM (Soc. Ind. Appl. Math.) J. Appl. Math. 39, 238 (1980).

${ }^{9}$ G. Bluman, SIAM (Soc. Ind. Appl. Math.) J. Appl. Math. 43, 1259 (1983).

${ }^{10}$ J. R. Ray, Phys. Rev. A 26, 729 (1982).

${ }^{11}$ R. S. Kaushal and D. Parashar, Phys. Rev. A 55, 2610 (1997).

${ }^{12}$ J. R. Burgan, M. R. Feix, E. Fijalkow, and A. Munier, Phys. Lett. A 74, 11 (1979).

${ }_{13}^{13}$ M. Boiti, F. Pempinelli, A. K. Pogrebkov, and M. C. Polivanov, Inverse Probl. 7, 43 (1991).

${ }^{14}$ V. G. Bagrov and B. F. Samsonov (preprint, quant-ph/9709040).

${ }^{15}$ V. Singh, S. N. Biswas, and K. Datta, Phys. Rev. D 18, 1901 (1978).

${ }^{16}$ A. V. Turbiner and A. G. Ushveridze, Phys. Lett. A 126, 181 (1987).

${ }^{17}$ A. González-López, N. Kamran, and P. J. Olver, Commun. Math. Phys. 153, 117 (1993).

${ }^{18}$ A. G. Ushveridze, Quasi-Exactly Solvable Models in Quantum Mechanics (IOP, Bristol, 1994).

${ }^{19}$ Note that Eq. (13) cannot in general be solved in closed form if $\chi_{1}$ is a polynomial in $x$ of degree $k>2$.

${ }^{20}$ P. Deift and E. Trubowitz, Commun. Pure Appl. Math. 32, 121 (1979).

${ }^{21}$ J. M. Sparenberg and D. Baye, J. Phys. A 28, 5079 (1995).

${ }^{22}$ Our choice of $Q_{n+1 / 2}$ differs from the one in Ref. 14 by the irrelevant constant factor $i^{n}$.

${ }^{23}$ If $n$ is even $Q_{n+1 / 2}$ is nonzero on the whole real axis.

${ }^{24}$ A comprehensive review of these methods is beyond the scope of this paper; see Refs. 26-30, and references therein for a detailed treatment.

${ }^{25}$ Some families of time-independent complex potentials which are quasi-exactly solvable have been recently proposed in the literature (Ref. 31).

${ }^{26}$ W. Magnus, Commun. Pure Appl. Math. 7, 649 (1954).

${ }^{27}$ J. Wei and E. Norman, J. Math. Phys. 4, 575 (1963).

${ }^{28}$ D. R. Truax, J. Math. Phys. 22, 1959 (1981).

${ }^{29}$ C. M. Cheng and P. C. W. Fung, J. Phys. A 21, 4115 (1988).

${ }^{30} \mathrm{~S}$. Zhang and F. Li, J. Phys. A 24, 6143 (1996).

${ }^{31}$ C. M. Bender and S. Boettcher (preprint, physics/9801007). 